\title{
Vertical-beam emittance correction with independent component analysis method
}

\author{
F. Wang and S. Y. Lee \\ Indiana University, Bloomington, Indiana 47405, USA
}

(Received 17 December 2007; published 15 May 2008)

\begin{abstract}
The vertical-beam emittance in an electron storage ring is mainly determined by two factors: the linear betatron coupling and the spurious vertical dispersion generated by magnet errors. We find that the contribution of spurious vertical dispersion is larger than that generated by the linear betatron coupling. Using the independent component analysis (ICA) method, we develop stop band corrections to reduce the vertical emittance. We demonstrate our method by making ICA and correction to a quadruple-bend achromatic low emittance lattice. Six families of skew quadrupoles can effectively minimize both the vertical dispersion and the linear betatron coupling.
\end{abstract}

DOI: 10.1103/PhysRevSTAB.11.050701

\section{INTRODUCTION}

In recent years, many low emittance electron storage rings around $3 \mathrm{GeV}$ beam energy have been proposed and constructed in the world. These storage rings are intended to produce a high brilliance $\mathrm{x}$ ray. Most of these low emittance lattices were designed based on the doublebend achromatic (DBA) cells, which are composed of two dipoles plus 5 or 6 pairs of matching quadrupoles to minimize the horizontal beam emittance. However, the achromatic condition is usually relaxed to further reduce the beam emittance by a factor of 3, called DBME (minimum emittance) lattices. Unfortunately, high field insertion devices (IDs) located in a dispersive straight section can spoil the beam emittances. A quadruple-bend achromatic (QBA) lattice concept has been proposed [1] to alleviate this difficulty.

A QBA-cell is a supercell made of 2 DBA cells, where 2 long (inner) and 2 short (outer) dipoles with matching quadrupoles are used to minimize the beam emittance [2]. For the same number of dipoles, quadrupoles, and straight sections, the emittance of a QBA-lattice is a factor of 2 smaller than that of a DBA-lattice. Although the nonachromatic DB-lattice provides a slightly smaller emittance than that of the corresponding QBA-lattice, the effective emittances of the bare DBME and QBA lattices are about equal [1]. When strong-field IDs are installed in the storage ring, the QBA lattice becomes superior to the nonachromatic DB lattice, particularly for beam energies lower than $4 \mathrm{GeV}$.

The photon brilliance of the synchrotron light source is inversely proportional to the product of the horizontal and vertical emittances. The vertical-beam emittance in a storage ring arises essentially from the linear betatron coupling and the quantum fluctuation due to the vertical dispersion function as derived in Ref. [3].

Systematic analysis and correction of the vertical emittance are needed to optimize the performance of electron storage rings. Measuring the vertical orbit shifts in many beam position monitor (BPM) locations has been success-
PACS numbers: 41.75.Ht, 41.85.-p, 52.59.-f, 29.27.Fh

fully used to reduce the vertical emittance by a factor of 6 [4]. Similarly, the orbit response matrix of the coupled motion has also been used to analyze and correct the vertical emittance [5]. Numerical simulations have been used to explore the correlation between the vertical emittance vs the $\left\langle D_{z}^{2}\right\rangle_{\mathrm{arc}}$, the average of the square of the vertical dispersion function in the dipole region [6].

In recent years, turn-by-turn BPM data are available in all high performance storage rings. The turn-by-turn data can be obtained in a single kick, or a single harmonic excitation by an $\mathrm{rf}$ dipole. Using the independent component analysis (ICA) [7], one can decompose the harmonic content of beam motion. Data analysis of turn-by-turn data at many BPMs will provide independent modes of particle motion. The turn-by-turn data of beam motion at all BPMs can usually be obtained in minutes, i.e., the measurement time is short. Isolating the BPM data in independent modes, one can obtain the betatron, synchrotron, synchrobetatron, and other modes. The time series of each individual mode is characterized by its spectrum, and the spatial function of each mode describes a component of the optical function of the accelerator. For example, the spatial function of a synchrotron mode is related to the dispersion function. Sometimes, the spatial function of an unexpected resonance mode can also be used to derive its resonance strength or stop band width.

Since a small vertical emittance is preferable in a high brilliance photon source, we need precise measurement and correction of betatron coupling and vertical dispersion function. This paper is intended to study the effectiveness of employing the ICA method to obtain the "derived" vertical dispersion function and examine the effectiveness of the vertical emittance correction. All of our BPM turnby-turn "data" are generated by the MAD tracking program. We add white noise into the BPM data, and use the ICA method to extract spatial functions of all independent modes. These spatial functions are called the derived betatron amplitude functions, dispersion functions, etc. We will study the effectiveness of the ICA method in determining the derived dispersion functions, and show that the fast- 
Fourier-transform spectra of the ICA derived vertical dispersion function agrees well with the theory based on stop band analysis. We then employ the stop band correction method with several families of skew quadrupole correctors. This method can correct the vertical dispersion function without identifying the source of errors. Since the vertical dispersion function is essentially generated by random errors, it would be very difficult to isolate each of these errors. Some physicists may prefer the $\chi^{2}$ minimization method to correct the vertical dispersion function and other physical quantities. Nevertheless, the stop band can be used to choose the best locations for correctors. The end results of these two methods are essentially identical.

Since the QBA-lattice has an advantage over the nonachromatic double-bend (DB) lattice in having zerodispersion straight sections and has an emittance smaller than that of a DBA-lattice, we demonstrate our correction method with a low emittance QBA-lattice. The QBA has another advantage over the DB nonachromatic lattice that the correction of the linear betatron coupling will not affect the vertical dispersion function. Naturally, the stop band correction method can also be implemented in DB lattices.

This paper will study the applicability of the stop band correction schemes in storage rings. We organize this paper as follows. In Sec. II, we compare the effect of the linear betatron coupling and the vertical dispersion function on the vertical emittance. In Sec. III, we examine the vertical dispersion function due to skew quadrupoles and dipole rolls, and discuss the accuracy of the ICA derived dispersion function. In Sec. IV, we discuss the stop bands for the linear betatron coupling and the vertical dispersion function. In Sec. V, we apply the stop band correction system for both the linear coupling and the vertical dispersionfunction correction. The conclusion is given in Sec. VI.

\section{VERTICAL-BEAM EMITTANCE}

The beam phase-space areas (emittances) of an electron storage ring arise from the equilibrium between quantum fluctuation and radiation damping. For a planer storage ring, the vertical-beam emittance arises essentially from the residual vertical dispersion function and the linear betatron coupling, where the vertical betatron motion is coupled to the horizontal betatron motion. Based on a Hamiltonian formalism, Guignard has developed an analytical treatment on the coupled betatron motions and its impact on the vertical emittance [3]. In the weak coupling approximation with $|G| \ll|\Delta|$, where $\Delta=\nu_{x}-\nu_{z}-l$ is the resonance proximity parameter, $\nu_{x}$ and $\nu_{z}$ are the betatron tunes, $l$ is the integer part of the difference between the betatron tunes, $G=|G| \exp (i \varphi)$ is the linear coupling resonance strength:

$$
G=\frac{1}{2 \pi B \rho} \oint \frac{\partial B_{x}}{\partial x} \sqrt{\beta_{x} \beta_{z}} e^{i\left(\psi_{x}-\psi_{z}-\Delta \cdot \theta\right)} d s,
$$

where $\psi_{x}$ and $\psi_{z}$ are the betatron phases and $\theta=s / R$ is the orbiting angle. Employing the Taylor expansion, we obtain the vertical emittance [3]:

$$
\begin{aligned}
\epsilon_{z} & =\epsilon_{d}+\epsilon_{c}+\epsilon_{d c}, \\
\epsilon_{d} & =\frac{C_{q} \gamma^{2}}{\mathcal{J}_{z} \rho}\left\langle\mathcal{H}_{z}\right\rangle, \\
\epsilon_{c} & =\frac{C_{q} \gamma^{2}}{\mathcal{J}_{x} \rho} \frac{|G|^{2}}{2 \Delta^{2}}\left\langle\mathcal{H}_{x}\right\rangle, \\
\epsilon_{d c} & =\frac{C_{q} \gamma^{2}}{\mathcal{J}_{z} \rho} \frac{|G|}{|\Delta|}\left\langle\operatorname{Re}\left[\left(\eta_{x}+i \zeta_{x}\right)\left(\eta_{z}-i \zeta_{z}\right) e^{i \phi}\right]\right\rangle,
\end{aligned}
$$

where $C_{q}=3.83 \times 10^{-13} \mathrm{~m}, \gamma$ is the Lorentz factor, $\rho$ is the dipole bending radius, $\mathcal{J}_{x}$ and $\mathcal{J}_{z}$ are the horizontal and vertical damping partition numbers, $\phi=\psi_{x}-\psi_{z}-$ $\Delta \cdot \theta+\varphi$, the $\mathcal{H}$-functions are $\mathcal{H}_{x}=\eta_{x}^{2}+\zeta_{x}^{2}$ and $\mathcal{H}_{z}=\eta_{z}^{2}+\zeta_{z}^{2}$, and $\eta_{x, z}$ and $\zeta_{x, z}$ are the normalized horizontal and vertical dispersion functions:

$$
\begin{array}{ll}
\eta_{x}=\frac{D_{x}}{\sqrt{\beta_{x}}}, & \zeta_{x}=\frac{1}{\sqrt{\beta_{x}}}\left(\beta_{x} D_{x}^{\prime}+\alpha_{x} D_{x}\right), \\
\eta_{z}=\frac{D_{z}}{\sqrt{\beta_{z}}}, & \zeta_{z}=\frac{1}{\sqrt{\beta_{z}}}\left(\beta_{z} D_{z}^{\prime}+\alpha_{z} D_{z}\right) .
\end{array}
$$

The equilibrium vertical emittance $\epsilon_{z}$ consists of the three terms: the first term $\epsilon_{d}$ arising from the vertical dispersion, the second term $\epsilon_{c}$ of the coupling between the vertical and horizontal betatron oscillations, and the correlation between the horizontal and vertical dispersion functions $\epsilon_{d c}$. We carry out systematic numerical simulations with many random seeds to study the contributions of these three terms, and find that the correlation term between the horizontal and vertical dispersion functions is small (see Fig. 1 below). Neglecting the correlation term, the vertical emittance is

$$
\boldsymbol{\epsilon}_{z} \approx C_{q} \frac{\gamma^{2}}{\mathcal{J}_{z} \rho}\left[\left\langle\mathcal{H}_{z}\right\rangle+\frac{|G|^{2}}{2 \Delta^{2}} \frac{\mathcal{J}_{z}}{\mathcal{J}_{x}}\left\langle\mathcal{H}_{x}\right\rangle\right] .
$$

Here the equilibrium emittances are determined by the coupling of the vertical and horizontal betatron oscillations and the spurious vertical dispersion function generated by magnet errors. These two contributions are independent and uncorrelated, and the final emittance is the sum of them. Since the horizontal and vertical damping partition numbers are approximately equal to 1 , the equilibrium emittance ratio is

$$
\frac{\epsilon_{z}}{\epsilon_{x}}=\frac{\left\langle\mathcal{H}_{z}\right\rangle}{\left\langle\mathcal{H}_{x}\right\rangle}+\frac{|G|^{2}}{2 \Delta^{2}} .
$$

Among the expected sources of vertical emittance in accelerator lattices, the random rolls of quadrupoles and dipoles are most important. Quadrupole rolls couple the horizontal $x$ and the vertical $z$ planes. Figure 1 shows the three vertical emittance contributions generated by all 


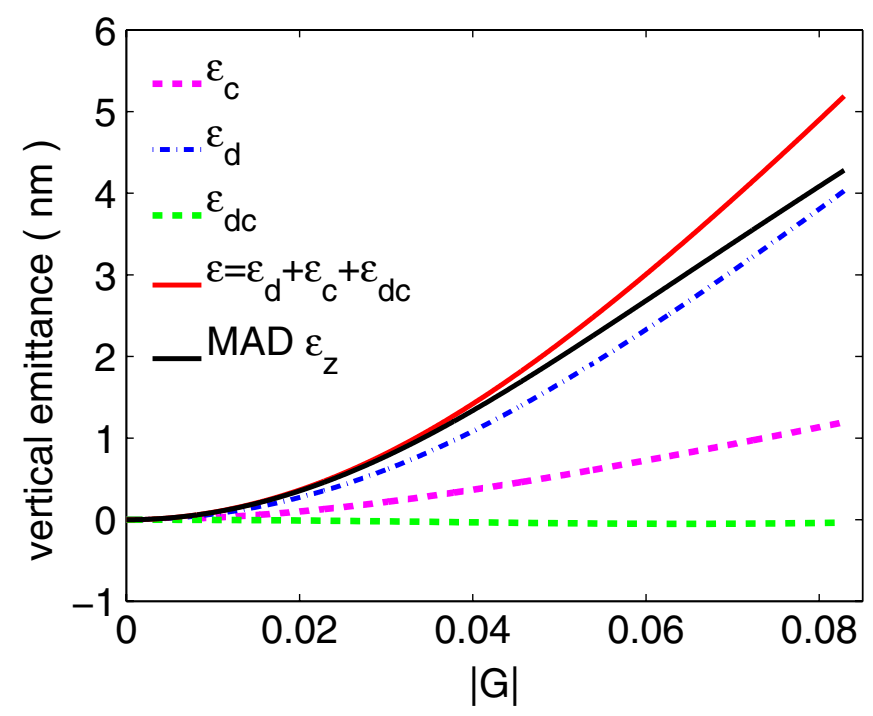

FIG. 1. (Color) The dispersion contribution $\epsilon_{d}$, the coupling contribution $\epsilon_{c}$, and the correlation $\epsilon_{d c}$ for a low emittance QBA-lattice [1] are plotted vs the linear coupling strength $G$. The linear coupling strength is varied by a random roll of all quadrupoles in uniform distribution. Note that the correlation term is negligible. The analytical solution $\epsilon$ agrees well with that obtained in the MAD modeling for $|G| \leq 0.05$, where the betatron functions are not strongly perturbed.

quadrupoles with random rolls with uniform distribution vs the coupling strength $G$ for a low emittance lattice [1].

The contribution from correlation term $\epsilon_{d c}$ is small. The vertical emittance is dominated by the vertical dispersion $\epsilon_{d}$ and the betatron coupling $\epsilon_{c}$. In the full coupling condition, the theory developed above allows one to esti-

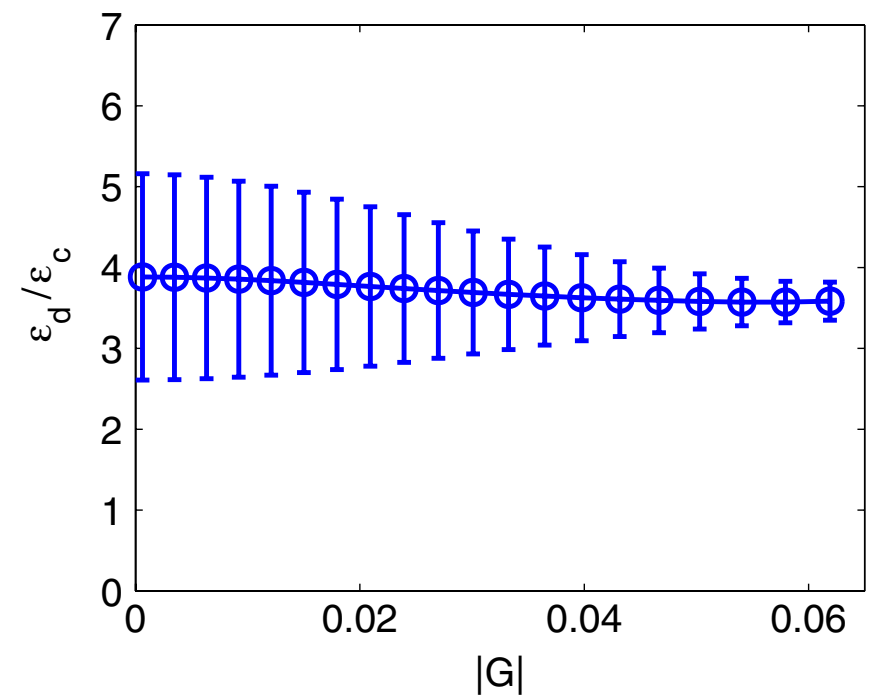

FIG. 2. (Color) The vertical emittance arises essentially from the vertical dispersion $\epsilon_{d}$ and the betatron coupling $\epsilon_{c}$. Our numerical simulations for a QBA-lattice show that their ratio $\epsilon_{d} / \epsilon_{c}$ is about four. This ratio depends on lattices to be discussed in the Appendix. mate the emittance ratio, which can be reached for different amplitudes of the vertical dispersion. The analytical solution $\epsilon_{z}=\epsilon_{d}+\epsilon_{c}+\epsilon_{d c}$ in Fig. 1 agrees very well with the MAD modeling based on the QBA-lattice. The analytical result is good approximation up to the linear betatron-coupling coefficient $G$ of 0.05 .

The ratio $\epsilon_{d} / \epsilon_{c}$ is shown in Fig. 2, where the error bar shows the variation of 10 random seeds used in the calculation. We note that $\epsilon_{d}$ is roughly 4 times as large as $\epsilon_{c}$ for the QBA-lattice, which has a relatively large $D_{x}$. When the parameter $G$ is small, the ratio is more sensitive to the dispersion-function stop band width to be discussed in the next section. In the Appendix, we compare the dependence of the ratio $\epsilon_{d} / \epsilon_{c}$ for different lattices.

\section{VERTICAL DISPERSION DUE TO RESIDUAL IMPERFECTIONS}

Since $\epsilon_{d}>\epsilon_{c}$, we need to examine some possible methods of vertical dispersion correction. The equation of motion for the vertical betatron motion is [8]

$$
z^{\prime \prime}+\frac{K_{z}(s)}{1+\delta} z=-\frac{\Delta B_{x}}{B \rho(1+\delta)},
$$

where $\delta=\left(p-p_{0}\right) / p_{0}$ is the fractional off-momentum deviation, $K_{z}(s)$ is the focusing function, $B \rho$ is the momentum rigidity of the on-momentum particle, and

$$
\frac{\Delta B_{x}}{B \rho}=\frac{1}{\rho}\left(a_{0}+b_{1} z+a_{1} x+2 b_{2} x z+\cdots\right) .
$$

Here $a_{0}$ arises essentially from the dipole roll, $b_{1}$ is the gradient error, $a_{1}$ is the skew quadrupole field, and $b_{2}$ is the sextupole field. Substituting $x=x_{\mathrm{co}}+D_{x} \delta+x_{\beta}$, we obtain

$$
\begin{aligned}
z^{\prime \prime}+\frac{\tilde{K}_{z}(s)}{1+\delta} z= & -\frac{1}{(1+\delta) \rho}\left[a_{0}+a_{1}\left(x_{\mathrm{co}}+x_{\beta}+D_{x} \delta\right)\right. \\
& \left.+2 b_{2}\left(x_{\beta}+D_{x} \delta\right) z\right]
\end{aligned}
$$

where the effective focusing function is $\tilde{K}_{z}(s)=K_{z}(s)+$ $b_{1} / \rho+2 b_{2} x_{\mathrm{co}}$. Expanding the vertical coordinate in $z=$ $z_{\mathrm{co}}+D_{z} \delta+z_{\beta}$, we find

$$
\begin{aligned}
z_{\mathrm{co}}^{\prime \prime}+\tilde{K}_{z}(s) z_{\mathrm{co}} & =-\frac{a_{0}}{\rho}-\frac{a_{1}}{\rho} x_{\mathrm{co}} \\
z_{\beta}+\tilde{K}_{z}(s) z_{\beta} & =-\frac{a_{1}+2 b_{2} z_{\mathrm{co}}}{\rho} x_{\beta}-\frac{2 b_{2}}{\rho} x_{\beta} z_{\beta} \\
D_{z}^{\prime \prime}+\tilde{K}_{z}(s) D_{z} & =h_{z}(s),
\end{aligned}
$$

where

$$
h_{z}(s)=\tilde{K}_{z} z_{\mathrm{co}}+\frac{a_{0}+a_{1} x_{\mathrm{co}}}{\rho}-\frac{a_{1}+2 b_{2} z_{\mathrm{co}}}{\rho} D_{x}(s) .
$$

Major sources of $h_{z}$ are vertical closed orbit error in quadrupoles $\tilde{K}_{z} z_{\text {co }}$, dipole roll $a_{0} / \rho$, and skew quadrupole field error in locations with a nonzero horizontal dispersion 
function. The skew quadrupole field produced by the quadrupole roll can produce both linear betatron coupling and vertical dispersion. In fact, the horizontal and vertical closed orbit, betatron functions, and dispersion functions are all coupled. When coupling is small and the horizontal betatron tune is sufficiently far away from an integer, the normalized vertical dispersion functions of Eq. (4) are

$$
\begin{gathered}
\eta_{z}=\frac{1}{2 \sin \pi \nu_{z}} \int_{s}^{s+C} \sqrt{\beta_{z}(t)} h_{z}(s) \cos \left[\Phi_{z}(s, t)\right] d t, \\
\xi_{z}=\frac{-1}{2 \sin \pi \nu_{z}} \int_{s}^{s+C} \sqrt{\beta_{z}(t)} h_{z}(s) \sin \left[\Phi_{z}(s, t)\right] d t,
\end{gathered}
$$

where $\Phi_{z}(s, t)=\pi \nu_{z}+\psi_{z}(s)-\psi_{z}(t)$ is the phase function, $\nu_{z}$ is the vertical betatron tune, and $\psi_{z}$ is the vertical betatron phase function. The dispersion functions are obtained by closed orbit integrals.

\section{A. The ICA method}

The ICA method has recently been introduced to analyze beam motion in accelerators [7]. Using numerical algorithms to process the turn-by-turn data at many BPM positions, one can filter out white noise and attain independent components of the beam motion. Possible independent components of beam motion are the coupled betatron motion, synchrotron motion, and other dynamic sources. This method has been successfully applied to the Fermilab Booster [7].

If one assumes that the transfer function of the BPM system is linear, the data sampled by BPMs can be considered as a linear combination of source signals. The physical origin of ICA modes can be identified by their spatial and temporal functions. Since particle motion in accelerators is essentially harmonic, it is nature to use the

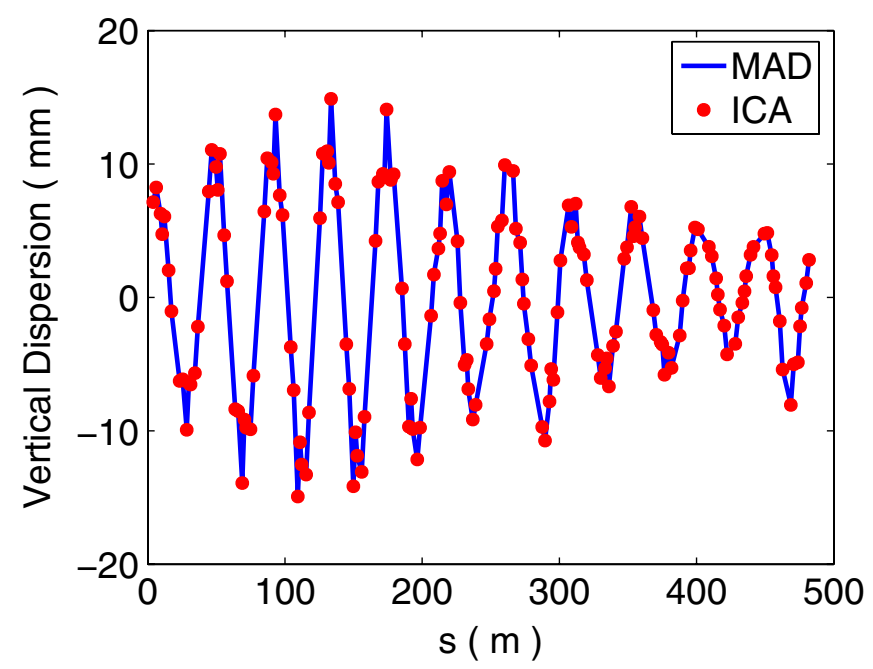

FIG. 3. (Color) The ICA derived dispersion function is compared with the MAD modeling based on a QBA low emittance lattice.

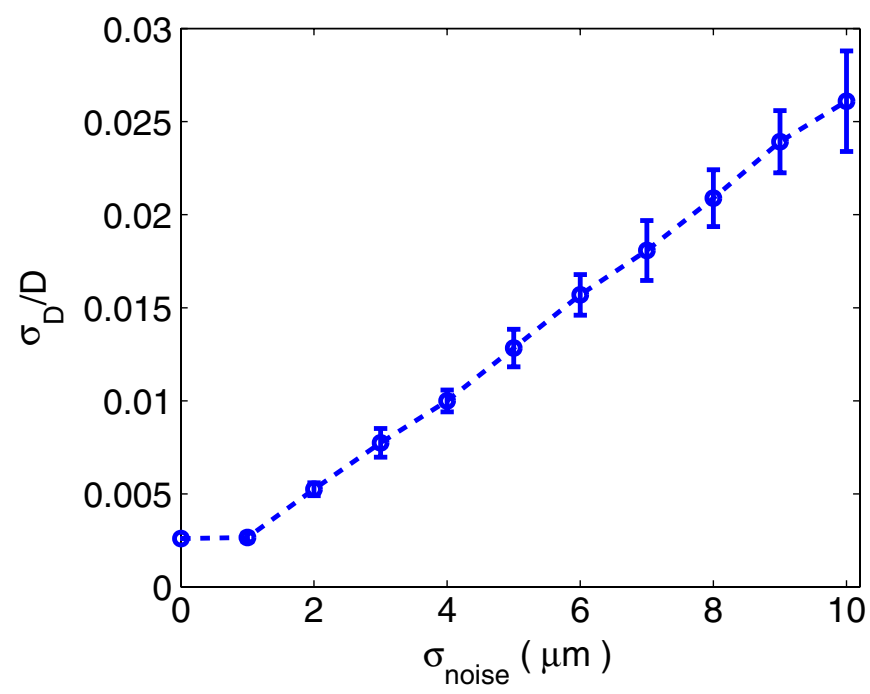

FIG. 4. (Color) Estimation of errors of ICA methods with various random noise levels. The estimation at each noise level $\sigma_{\text {noise }}$ of BPMs is made by repeating the ICA analysis of the vertical dispersion 20 times with Gaussian random noise added to each BPM.

time-correlation based ICA method, which is efficient in isolating the source signals without overlapping power spectra. The synchrotron mode, identified by the synchrotron tune, enables us to obtain the vertical dispersion function.

\section{B. The ICA derived dispersion function}

Using particle tracking data of 1024-turn, we find good agreement between the ICA derived dispersion function and simulation data as shown in Fig. 3, where the red dots are the derived vertical dispersion function at BPM locations and the blue curve is the vertical dispersion obtained from MAD modeling. The ICA derived dispersion function agrees with the vertical dispersion of MAD modeling to an rms error of $\sigma_{D} / D \approx 0.22 \%$.

In reality, BPM readings always contain random noise which limits the precision of the vertical dispersion to be analyzed by the ICA method. We insert white Gaussian noise into data sampled by BPMs. The rms errors of the resulting vertical dispersion function are shown in Fig. 4.

\section{THE INTEGER STOP BAND INTEGRALS}

As an example, we consider the effect of skew quadrupoles on the vertical dispersion in Eq. (8):

$$
D_{z}^{\prime \prime}+K_{z}(s) D_{z}=\frac{1}{B \rho} \frac{\partial B_{x}}{\partial x} D_{x}
$$

where $\frac{\partial B_{x}}{\partial x}$ is the skew quadrupole field strength and $D_{x}$ is the horizontal dispersion function at the skew quadrupole location. With the Floquet transformation to Eq. (12): 


$$
\eta_{z}=\frac{D_{z}}{\sqrt{\beta_{z}}}, \quad \phi=\frac{1}{\nu_{z}} \int_{0}^{s} \frac{d s}{\beta},
$$

the equation for the normalized coordinates becomes

$$
\frac{d^{2} \eta_{z}}{d^{2} \phi^{2}}+\nu_{z}^{2} \eta_{z}=\nu_{z}^{2} \beta_{z}^{3 / 2} \frac{1}{B \rho} \frac{\partial B_{x}}{\partial x} D_{x},
$$

where $\beta_{z}$ is the vertical betatron function at the skew quadrupole location. Since the driving function in the right-hand side of Eq. (14) is a periodic function of $2 \pi$ in $\phi$, we obtain

$$
F(\phi)=\nu_{z} \beta_{z}^{3 / 2} \frac{1}{B \rho} \frac{\partial B_{x}}{\partial x} D_{x}=\sum_{k=-\infty}^{\infty} f_{k} e^{i k \phi} .
$$

For the skew quadrupole arisen from the quadrupole roll, $\frac{1}{B \rho} \frac{\partial B_{x}}{\partial x}=K_{1} \sin \left(2 \theta_{\text {roll }}\right)$, where $K_{1}$ is the quadrupole strength, and $\theta_{\text {roll }}$ is the roll angle of the quadrupole. The Fourier amplitude $f_{k}$ is the integer stop band integral:

$$
f_{k}=\frac{1}{2 \pi} \oint \sqrt{\beta_{z}} D_{x} \frac{1}{B \rho} \frac{\partial B_{x}}{\partial x} e^{-i k \phi} d s .
$$

With the Fourier expansion, the vertical dispersion function becomes

$$
D_{z}(s)=\nu_{z} \sqrt{\beta_{z}(s)} \sum_{k=-\infty}^{\infty} \frac{f_{k} e^{i k \phi}}{\nu_{z}^{2}-k^{2}} .
$$

The vertical dispersion is most sensitive to the error harmonics close to the vertical betatron tune. The resulting vertical dispersion is usually dominated by a few harmon-

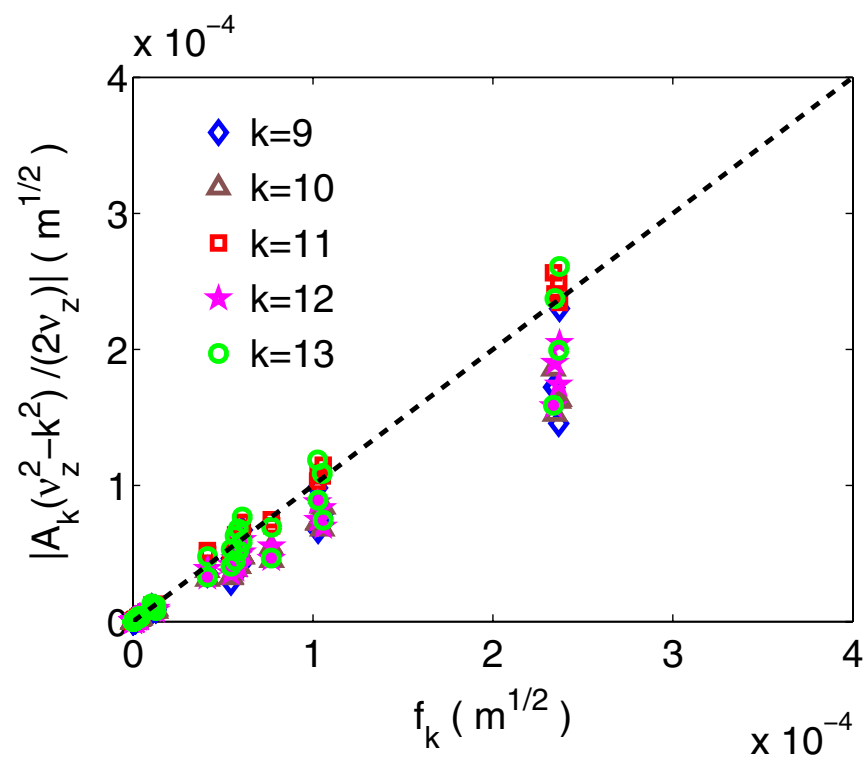

FIG. 5. (Color) Comparison of $A_{k}\left(\nu_{z}^{2}-k^{2}\right) / \nu_{z}$, where $A_{k}$ is the $k$ th Fourier harmonics of the dispersion function, for the QBAlattice [1], with $f_{k}$ of Eq. (16). The vertical dispersion functions are generated by $1 \mathrm{mrad}$ quadrupole roll to different quadrupoles in the QBA-lattice.

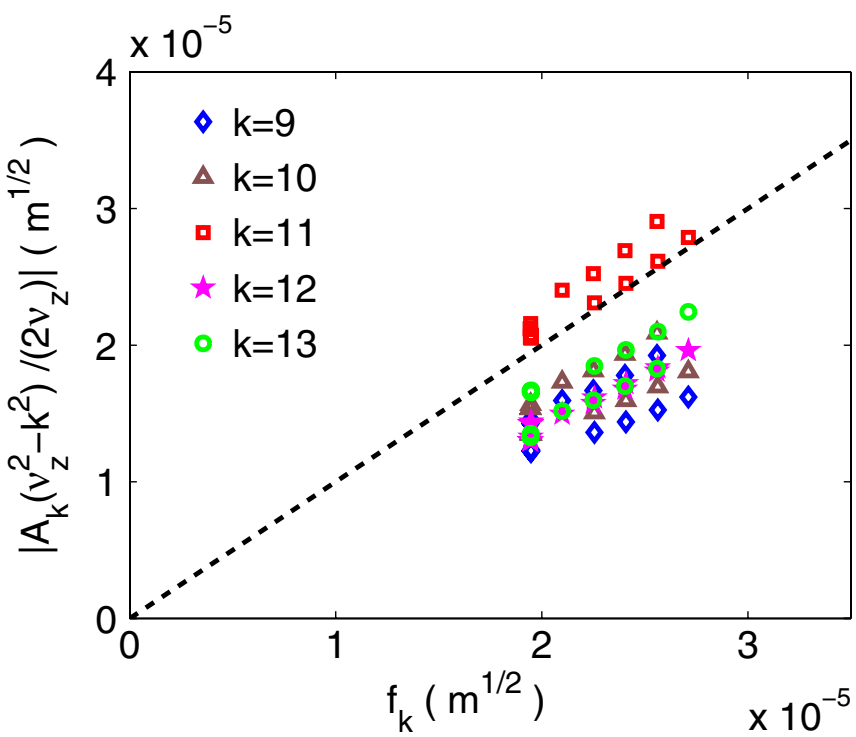

FIG. 6. (Color) Comparison of $A_{k}\left(\nu_{z}^{2}-k^{2}\right) / \nu_{z}$ derived from the vertical dispersion function with the Fourier harmonics $f_{k}$ of Eq. (18). The vertical dispersion function is generated by a $1 \mathrm{mrad}$ dipole roll to different dipoles in the QBA-lattice [1].

ics near $\left[\nu_{z}\right]$, which is an integer nearest to the betatron tune.

We carried out systematic stop band analysis for the low emittance QBA-lattice [1] by making quadrupole rolls on different quadrupoles. Figure 5 compares the stop band integrals derived from the Fourier analysis of the vertical dispersion function with those of Eq. (16).

In general, the vertical dispersion function can be produced by any horizontal dipole-field error $h_{z}(s)$ in Eq. (8). We can carry out Floquet transformation by changing $s$ to $\phi=\frac{1}{\nu_{z}} \int_{0}^{s} \frac{1}{\beta_{z}} d s$, and define the Fourier harmonics $f_{k}$ of the perturbation as

$$
f_{k}=\frac{1}{2 \pi} \oint \sqrt{\beta_{z}} h_{z}(s) e^{-i k \phi} d s,
$$

where $k$ is an integer. We also carry out simulations with dipole errors. Figure 6 compares the $A_{k}\left(\nu_{z}^{2}-k^{2}\right) / \nu_{z}$, where $A_{k}$ is the Fourier harmonic of the vertical dispersion function, with $f_{k}$ of Eq. (18) for a $1 \mathrm{mrad}$ dipole roll for the QBA-lattice [1].

\section{CORRECTION SCHEME}

Since the vertical dispersion function plays a major role in the vertical emittance for synchrotron light source and damping rings of linear colliders, it is important to be able to correct the spurious vertical dispersion. This allows one to maximize the beam brilliance. Figures 5 and 6 show that the dispersion function can be expanded in terms of major stop band integrals. An effective method for the dispersionfunction correction is to eliminate the largest components of the stop band integral with harmonics near the vertical 


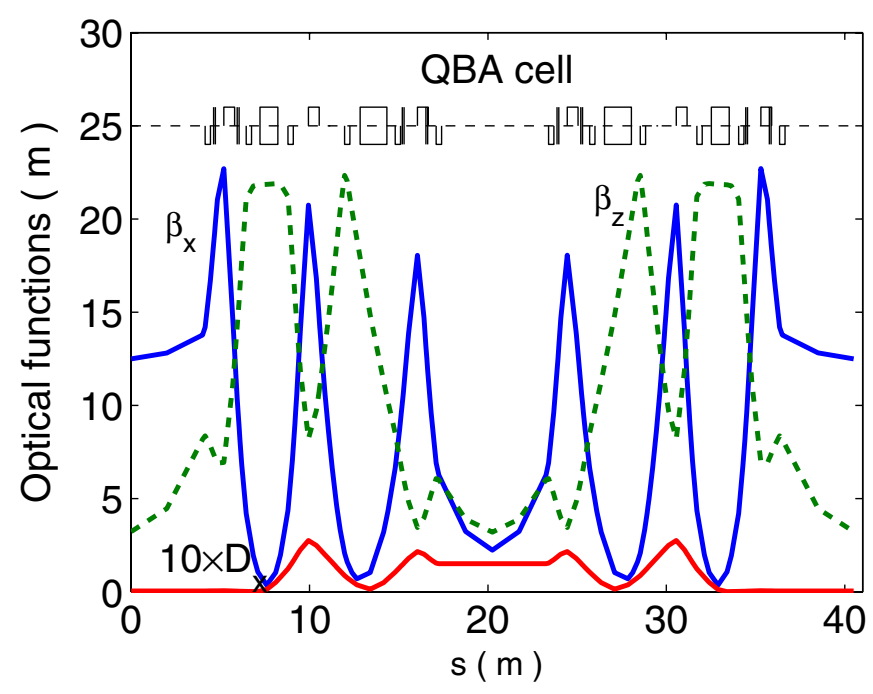

FIG. 7. (Color) Optical functions for a section of a quadruplebend achromatic lattice [1].

betatron tune. To correct these stop bands, we use skew quadrupoles to compensate all stop bands without altering the betatron tunes or introducing any unwanted harmonics.

To provide a concrete example for the stop band correction, we study a QBA-lattice with a circumference of $486 \mathrm{~m}$ and 12 QBA cells for $3 \mathrm{GeV}$ beam energy [1]. There are ten quadrupole families with reflection symmetry in a superperiod. The betatron tunes are $\nu_{x}=26.25$ and $\nu_{z}=11.37$, and the natural chromaticities are -64 and -30 . Figure 7 shows the optical functions. The emittance of this lattice is $2.7 \mathrm{~nm}$ rad. The effective emittance of the QBA-lattice is about a factor of 2 smaller than that of the corresponding DBA-lattice, while keeping some zerodispersion sections for high field damping wigglers. High field wavelength shifters located in the dispersive free straight section can further decrease the beam emittance.

\section{A. The estimation of correction requirement}

We would like to know the typical size of the stop band integrals. Using the QBA-lattice [1] example with random rolls in quadrupoles and dipoles at $1 \mathrm{mrad}$ amplitude in uniform distribution, the typical stop band integrals are listed in Table I. In practice, the perturbing field errors, due mainly to random rotation errors in the dipole magnets and the quadrupoles, are not known a priori. Figure 8 shows the vertical dispersion function generated by all quadrupoles, where the tilt angles vary between $-1 \mathrm{mrad}$ and $1 \mathrm{mrad}$ with a uniform random distribution.

TABLE I. Estimation of stop band integrals.

\begin{tabular}{lrr}
\hline \hline Driving terms & \multicolumn{1}{c}{$\operatorname{Re}$} & \multicolumn{1}{c}{$\operatorname{Im}$} \\
\hline$f_{11}\left(\mathrm{~m}^{1 / 2}\right)$ & $-3.228 \times 10^{-4}$ & $2.909 \times 10^{-4}$ \\
$f_{12}\left(\mathrm{~m}^{1 / 2}\right)$ & $1.709 \times 10^{-4}$ & $-3.604 \times 10^{-4}$ \\
$G$ & $2.200 \times 10^{-3}$ & $2.119 \times 10^{-4}$ \\
\hline \hline
\end{tabular}

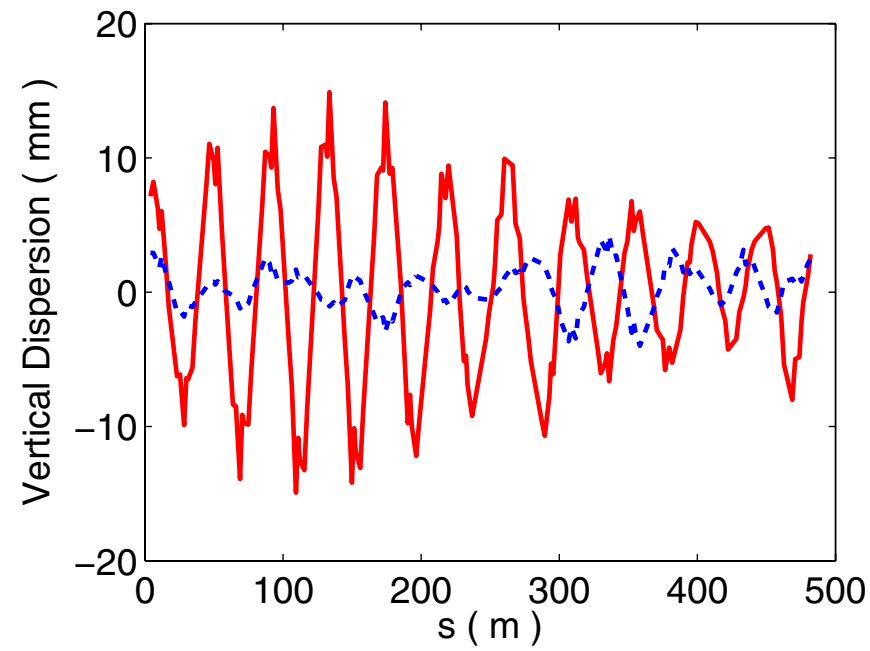

FIG. 8. (Color) The vertical dispersion generated by all quadrupoles with a uniform randomly distributed tilt angle $\pm 1 \mathrm{mrad}$ before (red solid line) and after (blue dashed line) harmonic correction.

Using the ICA analysis for the BPM turn-by-turn data, we obtained the derived vertical dispersion, and evaluate the stop band integrals. Similarly, we can determine the linear coupling resonance strength by measuring the minimum split between two betatron tunes or by using the turnby-turn data to construct the coupled phase-space tori [9]. The resonance strengths for the vertical dispersion functions for random quadrupole and dipole rolls of $\pm 1 \mathrm{mrad}$ in uniform distribution are given in Table I. The stop band strengths depend on the distribution of the skew quadrupole errors.

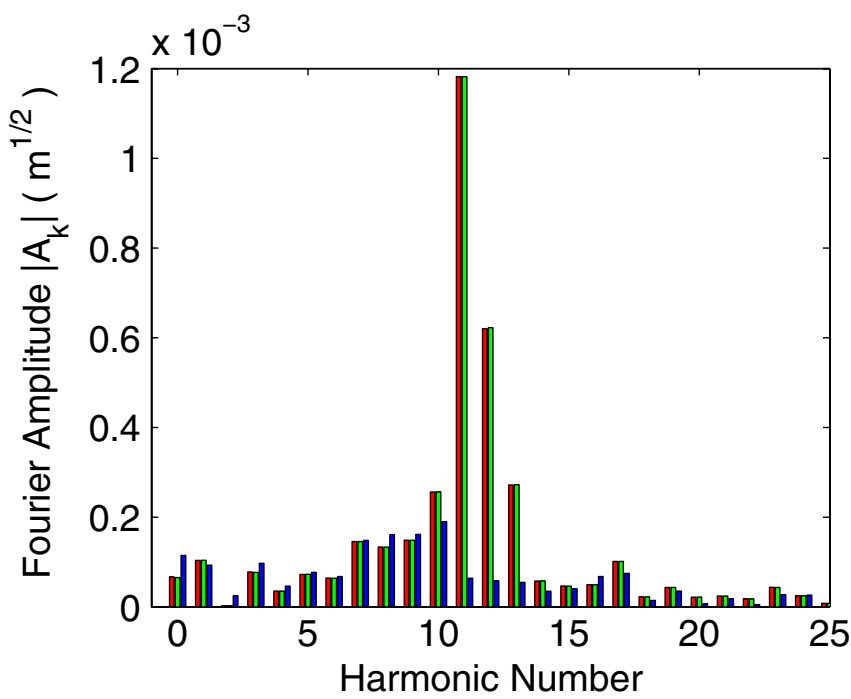

FIG. 9. (Color) Fourier harmonics of the normalized vertical dispersion function before (red bars) and after (blue bars) stop band correction show that the harmonic correction is effective in reducing the vertical dispersion. The linear betatron-coupling correction does not alter the Fourier harmonics of the vertical dispersion function (green bars). 
The vertical dispersion function produced by the horizontal field errors has a few dominant harmonics near the vertical betatron tune of 11.37. Figure 9 shows the amplitudes of Fourier harmonics of the vertical dispersion function. We note that the dominant harmonics are 11 and 12. If we want to correct two harmonics, we need four families of skew quadrupoles with proper phase relation.

\section{B. Excitation scheme}

Based on what we have discussed, the vertical emittance correction can be accomplished by correction to the linear betatron coupling and the vertical dispersion function. We would like to demonstrate the correction scheme by using 6 families of skew quadrupoles with a total of 38 skew quadrupoles distributed around the QBA ring.

If we place the betatron-coupling correctors in dispersion-free straight sections of QBA cells, these correctors will not affect the vertical dispersion function. Two families of betatron motion coupling correctors, each with 3 units, are thus placed in the dispersion-free sections. Detailed analysis of QBA-lattice shows that the phase difference of two skew quadrupole families is $\psi_{x}-\psi_{z}-$ $\left(\nu_{x}-\nu_{z}-\ell \theta\right) \approx 80.5^{\circ}$. i.e. they are nearly orthogonal and can be used to correct the real and imaginary part of the linear betatron coupling.

We arrange the other 32 skew quadrupole correctors into four families to correct the stop band integrals of harmonics 11 and 12. These skew quadrupoles are placed at straight sections in the dispersive region of QBA cells. We can attain a phase difference of nearly $90^{\circ}$ at the triplet and doublet quadrupole sections.

\section{Linear betatron-coupling correction}

We define the integrated skew quadrupole strength in thin lens approximation as

$$
Q=\frac{1}{2 \pi B \rho} \int \frac{\partial B_{x}}{\partial x} d s=\frac{\frac{\partial B_{x}}{\partial x} \ell_{s q}}{2 \pi B \rho},
$$

where $\ell_{s q}$ is the length of the skew quadrupole corrector. Using Eq. (1) and the QBA-lattice, we find

$$
\left(\begin{array}{l}
\operatorname{Im} G \\
\operatorname{Re} G
\end{array}\right)=\left(\begin{array}{ll}
-4.359 & -0.808 \\
-0.684 & -4.241
\end{array}\right)\left(\begin{array}{l}
Q_{1} \\
Q_{2}
\end{array}\right) .
$$

Here the unit of the $Q_{i}$ is $\mathrm{m}^{-1}$, and the unit of the matrix element is in $\mathrm{m}$. The phase difference between two families of linear coupling correctors is near an odd-integer multiple of $90^{\circ}$; these two families can effectively compensate the linear betatron-coupling resonance. Since these coupling correctors are located at the zero-dispersion straight sections, these correctors do not affect the vertical dispersion function in linear order. After the coupling correction, the remaining contribution to the vertical emittance comes from quantum fluctuation in the presence of the vertical dispersion function.

\section{Stop band correction for the vertical dispersion function}

In order to minimize quantum excitation in the vertical plane and to minimize the vertical-beam size, we need to reduce the vertical dispersion function. As an example, we consider four families of the skew quadrupoles to correct the vertical dispersion function. We use a harmonic correction scheme by correcting two major harmonics nearest to the vertical betatron tune. In our QBA-lattice example, we consider the harmonics 11 and 12 .

The skew quadrupoles introduced to correct the vertical dispersion function may also change the linear betatroncoupling strength. Thus we must simultaneously include the linear betatron coupling as a correction package, i.e., 6 families of skew quadrupoles. The coupling and the vertical dispersion stop band widths are linear functions of the skew quadrupole strengths:

$$
\left(\begin{array}{l}
\operatorname{Im} G \\
\operatorname{Re} G \\
\operatorname{Im} f_{11} \\
\operatorname{Re} f_{11} \\
\operatorname{Im} f_{12} \\
\operatorname{Re} f_{12}
\end{array}\right)=M\left(\begin{array}{l}
Q_{1} \\
Q_{2} \\
Q_{3} \\
Q_{4} \\
Q_{5} \\
Q_{6}
\end{array}\right)
$$

where the $M$ matrix for the QBA-lattice example is

$$
\left(\begin{array}{cccccc}
-4.359 & -0.808 & 1.439 & 2.623 & -2.380 & -0.279 \\
-0.684 & -4.241 & 5.140 & 4.850 & -3.552 & 0.840 \\
-0.004 & 0.002 & -1.157 & 0.162 & 0.004 & -0.002 \\
-0.000 & -0.008 & 0.162 & 1.155 & -0.019 & -0.000 \\
0.053 & -0.044 & -0.057 & -0.064 & -9.140 & -0.004 \\
0.023 & 0.018 & 0.153 & 0.160 & 0.679 & -3.255
\end{array}\right) .
$$

The lower left matrix elements of $M$ are small, indicating that the correctors are very much independent for the harmonics 11 and 12. The diagonal matrix elements are large and thus the correction is very effective. The offdiagonal matrix elements in the upper right corner of the first two rolls are relatively large. This means that the skew quadrupoles for stop band correction can introduce sizable linear betatron coupling. Since the strengths of skew quadrupole correctors are relatively small, the linear coupling induced by the skew stop band correctors can easily be compensated by the coupling resonance correctors. Thus these 6 families of skew quadrupoles are acceptable.

\section{Simulation results}

The operation of the correction systems is simulated using the MAD program. The stop band integrals with harmonic numbers 11 and 12 were evaluated and used to obtain the required corrector strength. When the correctors are powered, the linear coupling correction has less impact on the vertical emittance than the stop band correction of the vertical dispersion function. The stop band correction 


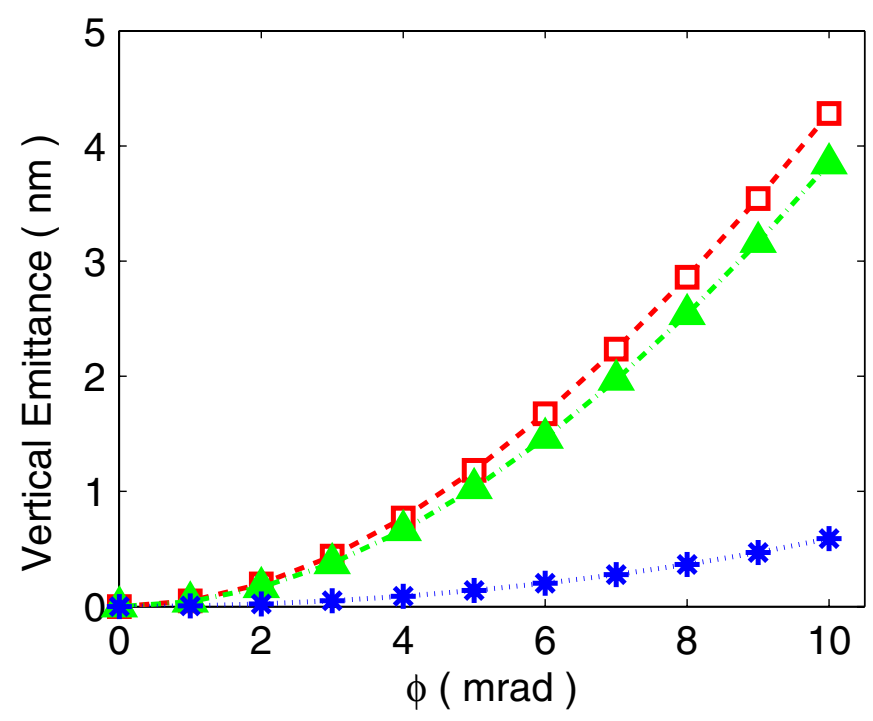

FIG. 10. (Color) The vertical emittance before (red line) and after (blue line) harmonic correction. For this random seed, the vertical emittance arises mainly from the vertical dispersion function, and thus the coupling correction (green line) has a little effect on the vertical emittance.

can reduce the equilibrium emittances $\epsilon_{z}$ by the factor of 7 as shown in Fig. 10.

The maximum value of vertical dispersion $D_{z, \max }$ was reduced from 15.5 to $4.2 \mathrm{~mm}$ shown in Fig. 8. To provide further reduction, one needs more skew quadrupole families. The above results could be explained in terms of the harmonics of the dispersion. Figure 9 shows the harmonics of the vertical dispersion before dispersion correction for the QBA lattices, where the harmonics near 11 have large amplitudes. After the stop band correction is applied, the two largest dominant harmonics were dramatically reduced. The coupling strength of the difference resonance can also be corrected simultaneously without altering the machine tunes or introducing any unwanted harmonics.

\section{CONCLUSION}

We have carried out a detailed evaluation of the source of the vertical emittance, and have studied a stop band correction scheme for the linear betatron coupling and the vertical dispersion function. We use the QBA-lattice as an example for the demonstration of this correction scheme. This scheme we have adapted is to adjust the skew quadrupole strengths to minimize the vertical dispersion produced by residual imperfections, as well as the coupling strength. In this manner, we have reduced the vertical emittance down to $5 \mathrm{pm}$, corresponding to a vertical to horizontal emittance ratio of less than $0.1 \%$. The direct stop band matrix is calculated from the model of the QBAlattice. Simulations confirmed that the stop band correction system has the strength and flexibility to cope with the expected magnet errors.

\section{ACKNOWLEDGMENTS}

This work is supported in part by grants from the U.S. Department of Energy DE-FG0292ER40747 and the National Science Foundation NSF PHY-0552389.

\section{APPENDIX: DEPENDENCE OF THE VERTICAL EMITTANCE ON LATTICES}

The vertical emittance generated by the vertical dispersion and the linear betatron coupling may vary from the various low emittance lattice configurations. In this Appendix, we analyze a few of these lattices. First, the linear coupling depends on the separation of the bare betatron tunes, i.e., the parameter $\Delta=\left|\nu_{x}-\nu_{z}\right|$. Second, the contribution from the vertical dispersion function depends on the value of the horizontal dispersion at the skew quadrupole locations. We have found that the stop bands can give an excellent description of the resulting vertical dispersion function. Thus, the actual contribution of the vertical emittance may depend on the design of various lattices.

To compare with the QBA-lattice discussed in this paper, we analyze the 18-cell DBA SPEAR3 lattice [10] and a 24cell DB-lattice with achromatic and the minimum emittance configurations [11]. The natural emittance of low emittance lattice scales as $(1 / \text { number of cells })^{3}$, while the values of the maximum horizontal dispersion function scales as $L \times(1 /$ number of cells $)$, where $L$ is the length of the dipole magnet. For these 4 lattices, we find the maximum dispersion function ranks as follows: $\hat{D}_{\text {SPEAR3 }}>$ $\hat{D}_{\mathrm{QBA}}>\hat{D}_{\mathrm{DBA}} \approx \sqrt{3} \hat{D}_{\mathrm{DBME}}$.

Since the effect of quadrupole roll is much larger than the corresponding dipole roll, we study only the effect of quadrupole roll. We carry out calculations with 10 random seeds. For each random seed, we calculate the linear

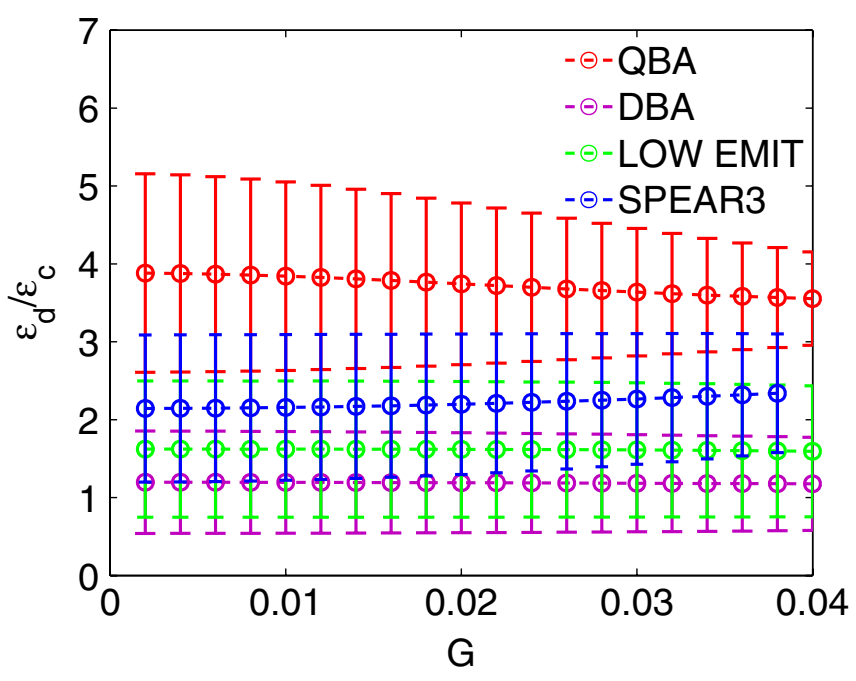

FIG. 11. (Color) Comparison of $\epsilon_{d} / \epsilon_{c}$ for a 24-cell QBA-lattice, the SPEAR 18-cell DBA-lattice and another 24-cell DBA and DBME lattices. 


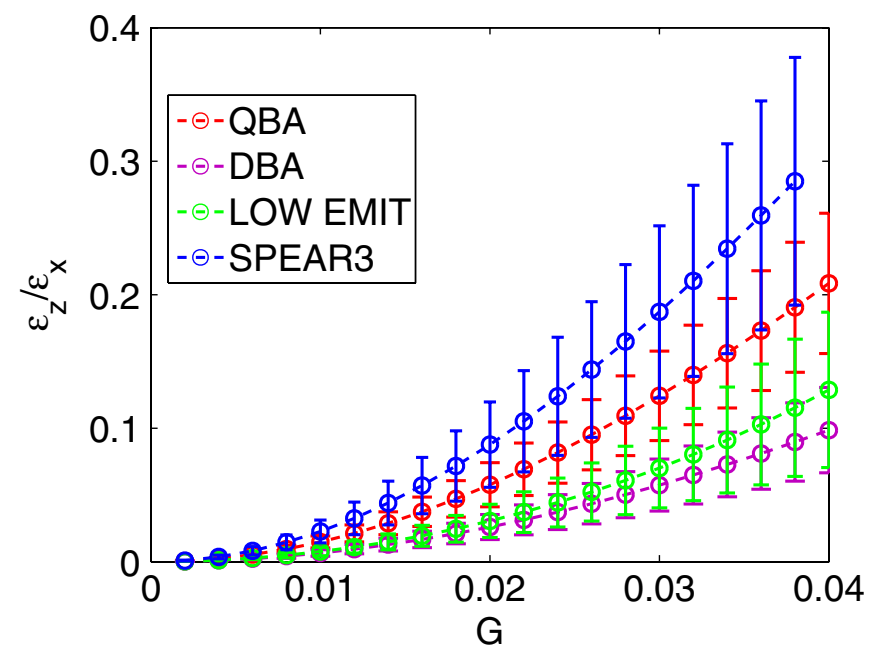

FIG. 12. (Color) The ratio of $\epsilon_{z} / \epsilon_{x}$ is plotted vs the linear coupling resonance strength $G$, used as a scaling parameter, for four low emittance lattices.

betatron-coupling parameter $G$, and change the overall strength of the quadrupole roll to vary the parameter $G$. Figure 11 shows the ratio of the contributions from the vertical dispersion function and the contribution from the linear betatron coupling.

We note that the $\epsilon_{d} / \epsilon_{c}$ ratio varies from 1 to 4 , presumably depending on the value of the horizontal dispersion functions. On the other hand, the actual ratios of the vertical emittance to the horizontal emittance, given by Eq. (5), depend also on the lattice types. Figure 12 shows $\epsilon_{z} / \epsilon_{x}$ vs $G$. We note that this ratio increases with the horizontal dispersion.

[1] M. H. Wang et al., Rev. Sci. Instrum. 78, 055109 (2007).

[2] S. Y. Lee, Phys. Rev. E 54, 1940 (1996).

[3] G. Guignard, Phys. Rev. E 51, 6104 (1995).

[4] J. Safranek and S. Krinsky, Proceedings of the Particle Accelerator Conference, Washington, DC, 1993 (IEEE, New York, 1993), p. 1491.

[5] C. C. Kuo et al., Proceedings of the Particle Accelerator Conference, Portland, OR, 2003 (IEEE, New York, 2003), p. 890; C. Steier et al., ibid., p. 3213.

[6] K. Kubo et al., Proceedings of the European Particle Accelerator Conference, Vienna, 2000 (EPS, Geneva, 2000), p. 483.

[7] X. Huang, S. Y. Lee, E. Prebys, and R. Tomlin, Phys. Rev. ST Accel. Beams 8, 064001 (2005).

[8] S.Y. Lee, Accelerator Physics (World Scientific, Singapore, 2004), 2nd ed.

[9] J. Y. Liu et al., Phys. Rev. E 49, 2347 (1994).

[10] X. Huang (private communications).

[11] C.-C. Kuo, H.-P. Chang, C.-T. Chen, G.-H. Luo, H.-J. Tsai, and M.-H. Wang, Proceedings of the Particle Accelerator Conference 2005 (IEEE, New York, 2005), p. 2989. 